\title{
Criptosporidiose em animais domésticos: aspectos epidemiológicos
}

\section{Cryptosporidiosis in livestock and pets: epidemiological aspects}

\author{
Katia Denise Saraiva Bresciani ${ }^{1 *}$; Monally Conceição Costa de Aquino²; \\ Anaiza Simão Zucatto²; Sandra Valéria Inácio ${ }^{3}$; Luiz da Silveira Neto ${ }^{4}$; \\ Natália Marinho Dourado Coelho5; Willian Marinho Dourado Coelho ${ }^{6}$; \\ Roberta Lomonte Lemos de Brito ${ }^{4}$; Milena Araúz Viol ${ }^{7}$; \\ Marcelo Vasconcelos Meireles ${ }^{8}$
}

\section{Resumo}

Estudos referentes á criptosporidiose são essenciais, devido sua relevância em saúde pública e por sua patogenicidade em animais de produção e de companhia. Nos últimos 20 anos, houve uma rápida expansão das pesquisas envolvendo o gênero Cryptosporidium, em grande parte relacionada a estudos moleculares, propiciando a descrição de várias espécies, genótipos e subtipos do parasito. A caracterização molecular de isolados de diferentes origens (animal, humana e ambiental) tem sido amplamente usada com o intuito de investigar o potencial zoonótico deste protozoário. As formas de transmissão documentadas são de animais para o ser humano, de pessoa para pessoa, por meio de ingestão ou do uso de água destinada a lazer contaminada direta ou indiretamente com oocistos esporulados. A elevada taxa de animais naturalmente infectados e a susceptibilidade ao protozoário justificam a importância de se atentar para a ocorrência desta enfermidade. Assim, são demonstrados aspectos epidemiológicos desta zoonose em animais domésticos.

Palavras-chave: Cryptosporidium spp., ruminantes, equinos, caninos, felinos

\begin{abstract}
Studies related cryptosporidiosis will be essential, due to its relevance in public health and pathogenicity in pets and production animals. Over the past 20 years, there has been a rapid expansion of research involving the Cryptosporidium genus, largely related to molecular studies, providing a description of
\end{abstract}

${ }^{1}$ Prof adjunto, Universidade Estadual Paulista "Julio de Mesquita Filho", UNESP, Faculdade de Medicina Veterinária de Araçatuba FMVA, Araçatuba, SP. E-mail: bresciani@fmva.unesp.br

${ }^{2}$ Médica Veterinária, M.e pelo Curso de Pós-Graduação em Ciência Animal, UNESP/FMVA, Araçatuba, SP. E-mail: monallyaquino@hotmail.com; anaszucatto@hotmail.com

${ }^{3}$ Bióloga, Discente de Doutorado do Curso de Pós-Graduação em Ciência Animal, UNESP/FMVA, Araçatuba, SP. E-mail: sandra_ byol@yahoo.com

${ }^{4}$ Médicos Veterinários, Discentes de Doutorado do Curso de Pós-Graduação em Medicina Veterinária, UNESP, Faculdade de Ciências Agrárias e Veterinárias de Jaboticabal, FCAV, Jaboticabal, SP. E-mail: luiz.silveira@hotmail.com; rolomonte@hotmail. com

${ }^{5}$ Enfermeira, Discente de Doutorado do Curso de Pós-Graduação em Ciência Animal, UNESP/FMVA, Araçatuba, SP. E-mail: marinhodourado@hotmail.com

${ }^{6}$ Médico Veterinário, Pós-Doutorando do Dept ${ }^{\circ}$ de Apoio, Produção e Saúde Animal, UNESP/FMVA, Araçatuba, SP. E-mail: willianmarinho@hotmail.com

${ }^{7}$ Médica Veterinária, Discente de Doutorado do Curso de Pós-Graduação em Ciência Animal, UNESP/FMVA, Araçatuba, SP. E-mail: milenaviol@hotmail.com

${ }^{8}$ Prof. Adjunto, UNESP, FMVA, Araçatuba, SP. E-mail: marcelo@fmva.unesp.br

* Autor para correspondência 
various species, genotypes and subtypes of the parasite. The molecular characterization of isolates from different sources (human, animal and environmental) has been widely used in order to investigate the potential zoonotic of this protozoa. The documented transmission forms from animals to humans, from person to person, through water intake or water for the leisure that are directly or indirectly contaminated with sporulated oocysts. The high rate of animals naturally infected and the susceptibility by protozoan, justify the importance of attending to the occurrence of this disease. So are demonstrated epidemiological aspects of this zoonotic disease in domestic animals.

Key words: Cryptosporidium spp., ruminants, horses, dogs, cats

\section{Introdução}

Os protozoários do gênero Cryptosporidium são parasitos intracelulares obrigatórios, pertencentes ao filo Apicomplexa e capazes de se desenvolver nas microvilosidades das células epiteliais do trato gastrintestinalde hospedeiros vertebrados (XIAO et al., 2004; KARANIS; ALDEYARBI, 2011; CHALMERS; KATZER, 2013).

Com o advento da Síndrome da Imunodeficiência Adquirida (HIV) na década de 1980, relatos de infecções oportunistas foram associados ao Cryptosporidium spp. e este passou a ser considerado um importante patógeno em seres humanos (FAYER, 2010; SMITH; NICHOLS, 2010; CIELOSZYK et al., 2012). Este parasito foi incluído na Iniciativa das Doenças Negligenciadas da Organização Mundial da Saúde por sua estreita relação com a população de baixo poder aquisitivo, precárias condições de saneamento básico e da qualidade da água consumida (SAVIOLI; SMITH; THOMPSON, 2006; ASSIS et al., 2013).

A infecção humana pode resultar de transmissão zoonótica ou antroponótica (XIAO, 2010). Dessa forma, a caracterização molecular de isolados de diferentes origens (animal, humana e ambiental) tem sido amplamente usada com o intuito de investigar o potencial zoonótico das espécies ou genótipos do protozoário (XIAO; FAYER, 2008). Atualmente, 26 espécies de Cryptosporidium são reconhecidas, sendo nove capazes de infectar humanos (ASSIS et al., 2013; SHARMA et al., 2013; CHALMERS; KATZER, 2013), sendo que diversos grupos de subtipos e sub-genótipos de Cryptosporidium têm sido identificados, dos quais os subtipos Cryptosporidium parvum IIa e IId foram identificados como zoonóticos (PLUTZER; KARANIS, 2009; HELMY et al., 2013).

A importância da criptosporidiose em animais de produção é traduzida não só pelo potencial zoonótico de algumas espécies como também pela perda econômica que esta infecção promove (OLSON et al., 2004). O C. parvum é o enteropatógeno mais comumente encontrado durante as primeiras semanas de vida em ruminantes, sendo considerado importante na etiologia das síndromes diarreicas neonatais. O parasito frequentemente age sozinho, mas a doença é mais pronunciada quando outros agentes estão presentes (RADOSTITS et al., 2000; SILVERLÅS et al., 2013).

As formas de transmissão documentadas são de animais para o ser humano, de pessoa para pessoa e de animal para animal, por meio de ingestão de alimentos ou do uso de água destinada a lazer que são contaminados direta ou indiretamente com resíduos fecais com oocistos esporulados (SMITH et al., 2006; WIDMER et al., 2012; MAIKAI; BABA-ONOJA; ELISHA, 2013).

A patogenia e o quadro clínico da doença são influenciados por vários fatores que incluem entre eles idade, competência imunológica do indivíduo infectado e a associação com outros patógenos (RADOSTITS et al., 2000). A infecção por Cryptosporidium spp. causa inflamação e atrofia das vilosidades intestinais resultando em perda da superfície de absorção, desequilíbrio no transporte de nutrientes (THOMPSON; PALMER; O'HANDLEY, 2008) e consequentemente comprometimento na produtividade animal 
(DECUBELLIS; GRAHAM, 2013).

Neste estudo, principalmente serão abordados aspectos epidemiológicos da infecção por Cryptosporidium spp. em animais de produção e de estimação.

\section{Criptosporidiose em Bubalinos}

O primeiro relato de Cryptosporidium em bubalinos foi efetuado na Itália (CANESTRITROTTI; QUESADA, 1983). Este protozoário já foi observado em fezes desta espécie em países como Cuba (RODRÍGUEZ-DIEGO et al., 1991), Índia (DUBEY; FAYER; RAO, 1992; BHAT; JUYAL; SINGLA, 2012), Brasil (ARAÚJO et al., 1996; RIBEIRO et al., 2000), Espanha (GOMÉZCOUSO et al., 2005), Itália (CACCIÓ et al., 2007; CONDOLEO et al., 2007; RINALDI et al., 2007), Egito (EL-KHODERY; OSMAN, 2008), Bolívia (MANZANILLA, 2009) com a prevalência da infecção por Cryptosporidium spp. oscilando entre $9,4 \%$ a $48,2 \%$. No entanto, nesses trabalhos não houve determinação da espécie ou do genótipo do parasito.

Araújo et al. (1996) avaliaram a ocorrência da infecção por Cryptosporidium spp. em 120 amostras fecais de bubalinos da região do Amapá, Brasil, por meio da técnica de Sheather, constatando a presença de oocistos em 10\% (12/120) das amostras. A identificação da espécie foi baseada em critérios morfométricos, sendo visualizados oocistos de $C$. parvum e C. muris. Neste mesmo país, a prevalência deste parasito foi estudada em gado bovino e bubalino no Vale da Ribeira, São Paulo, Brasil (SPÓSITO FILHA; FUJII; REBOUCAS, 1998).

Em búfalos jovens, a diarreia foi considerada a mais importante causa de perdas econômicas por gastroenterites, no Egito (EL-GHARI et al., 1994), na Itália (GALIERO; CONSALVO; CARULLO, 1994) e no Brasil (RIBEIRO et al., 2000).

Ribeiro et al. (2000) verificaram presença de oocistos de C. parvum em búfalos, mais frequentemente na sexta semana de idade e com maior prevalência em animais sem sinais de distúrbio gastrointestinal, sugerindo que bezerros não sintomáticos são potenciais fontes de infecção. Ao contrário, El-Khodery e Osman (2008), observaram que a faixa etária mais susceptível é de um a 15 dias e que a diarreia está significativamente associada à infecção.

Por meio da técnica de ELISA, Rinaldi et al. (2007) verificaram a ocorrência de 19,8\% (35/177) de C. parvum (coproantígenos) em búfalos com idade entre dois e 60 dias de vida, provenientes de 47 fazendas da região central da Itália. Em estudo semelhante, Condoleo et al. (2007) constataram uma ocorrência de 14,7\% (51/347) de coproantígenos em animais assintomáticos com idade entre uma a nove semanas, em 90 propriedades rurais e observaram uma associação positiva entre a infecção e o número elevado de búfalos $(>100)$ na propriedade.

A análise molecular de Cryptosporidium spp. em búfalos foi pioneiramente realizada por GomézCouso et al. (2005), que observaram infecção em uma novilha bubalina. O diagnóstico foi feito com base no exame de imunofluorescência direta que permitiu a visualização de oocistos indistinguíveis de C. parvum. Posteriormente, a caracterização molecular revelou Cryptosporidium parvum genótipo "pig".

Outro estudo que utilizou a ferramenta molecular para identificação de espécies de Cryptosporidium spp. em búfalos, foi efetuado por Cacció et al. (2007), que demonstraram $14 \%(8 / 57)$ de positividade por meio do teste de ELISA. A investigação molecular de seis dessas amostras revelou 100\% de homologia com C. parvum, sugerindo que estes animais representam fonte de infecção deste parasito.

A maioria das pesquisas sobre Cryptosporidium em búfalos foram baseados na identificação morfológica de oocistos nas fezes ou por meio de métodos imunológicos. Embora ambas as técnicas possam fornecer dados sobre a prevalência generalizada deste parasito, somente a biologia 
molecular é capaz de identificar as espécies ou genótipos do referido gênero, o que é relevante do ponto de vista de saúde pública (FAYER, 2010).

Há poucos estudos relacionados à classificação molecular de Cryptosporidium em bubalinos, com relatos de identificação de C. parvum, Cryptosporidium "pig" genotype e C. ryanae (GOMÉZ-COUSO et al., 2005; CACCIÓ et al., 2007; FENG et al., 2012; VENU et al., 2012, AQUINO, 2012; AMER et al., 2013).

Em búfalos, C. ryanae foi observado apenas por Feng et al. (2012), no Nepal e por Venu et al. (2012) na Índia, sendo que no Brasil, foi descrito pela primeira vez por Aquino (2012), que também encontrou de maneira inédita, o genótipo Cryptosporidium sp. W20486 nessa espécie animal.

\section{Criptosporidiose em Caprinos}

A primeira evidência de infecção por Cryptosporidium spp. foi na Austrália com acometimento de um cabrito com duas semanas de idade, que veio a óbito após curto episódio diarreico (MASON; HARTLEY; TILT, 1981).

O protozoário Cryptosporidium é considerado como um dos principais patógenos entéricos em cabras (DELAFOSSE et al., 2006) e tem sido observado em vários países do mundo e apresenta prevalências variáveis, como: 28,5\% em Sri Lanka (NOORDEEN et al., 2000), 2,5\% na França (CASTRO-HERMIDA et al., 2005); 4,8\% no Brasil (BOMFIM et al., 2005); 67,7\% na Argentina (VENTURINI etal., 2006); 4,8\% na Zâmbia(GOMA et al., 2007), 35,8\% na Bélgica (GEURDEN et al., 2008) e 16,66\% no Brasil (COELHO, 2011).

A gastroenterite por Cryptosporidium, denominada Síndrome da Diarreia Neonatal (FOREYT, 1990; PUGH, 2004; GOMA et al., 2007), é consequência da interação entre produtos parasitários, que lesam a barreira epitelial e interferemnas respostasimunológicas einflamatórias do hospedeiro (THOMPSON; PALMER;
O'HANDLEY, 2008), com comprometimento da conversão alimentar; redução no ganho de peso vivo, diminuição da produção leiteira, com morbidade alta em surtos e ocasionalmente com desfecho fatal (FOREYT, 1990; VIEIRA et al., 1997), gerando prejuízo econômico considerável ao criador (VIEIRA, 1999, NOORDEEN et al., 2012).

Geurden et al. (2008) constataram, por meio da PCR com o gene da glicoproteína $60 \mathrm{kDa}$ em cabritos, os subgenótipos IIa15G2R1 e IIdA22G1, com maior e menor potencial zoonótico, respectivamente. Cryptosporidium parvum subgenótipo IIa15G2R1, que apresenta grande importância em saúde pública, foi concomitantemente encontrado em cabritos, bezerros e em um leitão que compartilhavam o mesmo ambiente (COELHO, 2011).

Além disso, caprinos assumem significado importante na transmissão do Cryptosporidium spp. para o ser humano, visto que pode atuar como reservatório de grupos zoonóticos de $C$. parvum (MONIS; THOMPSON, 2003; QUÍLEZ et al., 2008; XIAO; ENG, 2008, RIEUX et al., 2013).

\section{Criptosporidiose em Ovinos}

A infecção por Cryptosporidium spp. foi referida com ineditismo na espécie ovina na Austrália, em cordeiros de sete a 21 dias de idade, com diarreia (BARKER; CARBONELL, 1974). Este parasito é considerado particularmente importante em pequenos ruminantes, sendo associado à Síndrome da Diarréia Neonatal e à imunosupressão com altos níveis de morbidade (GOMA et al., 2007; COSENDEY et al., 2008).

A ocorrência de criptosporidiose em rebanhos ovinos foi descrita em várias regiões geográficas, com incidências variáveis: $10,1 \%$ na Polônia (MAJEWSKA et al., 2000), 59\% na Espanha (CAUSAPÉ et al., 2002), 77,4\% nos Estados Unidos (SANTÍN; TROUT; FAYER, 2007), 11,2\% na Tunísia (SOLTANE et al. 2007); 24,5\% na Austrália (YANG et al., 2009), também 25\% (SILVA, 2007), 
41\% (COSENDEY et al., 2008), 6,7\% (FÉRES et al., 2009), 0\% (SEVÁ et al., 2010), 1,6\% (FIUZA et al., 2011), 15\% (ZUCATTO, 2013) no Brasil e 13,7\% (IMRE et al., 2013) na Romênia.

Em Botucatu, São Paulo, Green, Amarante e Mascarini (2005) realizaram colheita de amostras fecais em um grupo de 20 ovelhas e seus respectivos filhotes no dia do nascimento e aos $4^{\circ}, 8^{\circ}, 16^{\circ}, 32^{\circ} \mathrm{e}$ $64^{\circ}$ dias pós-parto. As amostras foram examinadas por meio do método de entrífugo-sedimentação em éter, por esfregaços corados com auramina e pela técnica de Ziehl-Neelsen modificada. Oocistos de Cryptosporidium spp. foram verificados em 26,7\% dos cordeiros, que foram eliminados com maior frequência ao $4^{\circ}$ dia de vida e em $31,9 \%$ das mães principalmente no $4^{\circ}$ dia pós-parto.

Santín, Trout e Fayer (2007) em Maryland, Estados Unidos da América (EUA), colheram por três dias consecutivos, um total de 189 amostras fecais de 32 mães e de 31 de seus respectivos filhotes no $7^{\circ}, 14^{\circ}$ e $21^{\circ}$ dias de idade. Pela reação de imunofluorescência, a ocorrência de Cryptosporidium spp. foi de $9 \%$ e pela PCR, 30\%, sendo demonstrada a maior sensibilidade da técnica molecular. Na faixa etária de 21 dias de idade, foi observado um maior número de cordeiros (19/31) eliminando oocistos fecais deste protozoário. A caracterização molecular revelou C. parvum (2/189), Cryptosporidium genótipo cervídeo (48/189) e C. bovis-like. Ovinos são comumente infectados pelas principais espécies: Cryptosporidium parvum, Cryptosporidium xiaoi e Cryptosporidium ubiquitum (FAYER; SANTÍN, 2009).

Na região de Araçatuba, São Paulo, Féres et al. (2009) observaram ocorrência de 6,7\% (31/460) para Cryptosporidium spp. em amostras fecais de cordeiros com até 30 dias de idade, por meio da técnica de coloração negativa com verde Malaquita. Caracterização molecular das amostras positivas ao PCR demonstrou o genótipo cervídeo, C. parvum 18S rRNA tipo A e C. parvum 18S rRNA tipo B, todos com potencial zoonótico.
Na China, Wang et al. (2010), obtiveram três espécies de Cryptosporidium, pela PCR, sendo C. andersoni em ovelhas gestantes e no pós-parto (04/82); C. xiaoi em cordeiros durante e após desmame (04/82) e $C$. genótipo cervine em amostras de cordeiros e ovelhas (74/82).

Em 10 ovinoculturas no Rio de Janeiro, Fiuza et al. (2011) detectaram 1,6\% (2/90) de infecção por este parasito em cordeiros entre dois a seis meses de idade, com identificação molecular de C. ubiquitum. Distribuída no mundo todo, está última espécie foi isolada em seres humanos (ONG et al., 2002; XIAO, 2010), mas tem sido observada em cordeiros com menos de doze meses de idade (SANTÍN; TROUT; FAYER, 2007; YANG et al., 2009; ROBERTSON; GJERDE; FURUSETH HANSEN, 2010; WANG et al., 2010; SWEENY et al., 2011).

Em cordeiros, C. parvum foi encontrado na região de Araçatuba/SP, por Féres et al. (2009) e na Polônia por Majewska et al. (2000). Fiuza et al. (2011) detectaram infecção por C. ubiquitum, no estado do Rio de Janeiro/RJ e Shen et al. (2011) na China. Silva (2007) observou na cidade de Tupi-Paulista/SP, C. parvum, C. bovis, C. felis e $C$. genótipo cervine. Ainda no sul da Austrália, Sweeny et al. (2011), identificaram $C$. parvum, $C$. ubiquitum e C. xiaoi.

Infecções geneticamente confirmadas por $C$. xiaoi (eram designadas anteriormente como por C. bovis-like). Esta nova espécie foi nomeada em homenagem ao Dr. Lihua Xiao por suas contribuições na taxonomia e epidemiologia molecular do Cryptosporidium (FAYER; SANTÍN, 2009). Este parasito foi observado em ovinos nos EUA (SANTÍN; TROUT; FAYER, 2007); Espanha (NAVARRO-I-MARTINEZ et al., 2007); Tunísia (ELWIN; CHALMERS, 2008); Reino Unido (MUELLER-DOBLIES et al., 2008); China (WANG et al., 2010), Noruega (ROBERTSON; GJERDE; FURUSETH HANSEN, 2010) e Austrália (SWEENY et al., 2011).

Subtipagem de sete isolados de C. parvum, 
baseados na análise da sequência do gene GP60, revelou a presença de cinco diferentes subtipos (IIaA17G1R1, IIaA16G1R1, IIdA20G1, IIdA24G1 and IIdA22G2R1) pertencentes a duas famílias de subtipos zoonóticos (IIa and IId). Estes achados podem sugerir o potencial papel de cordeiros neonatos como fonte de criptosporidiose humana (IMRE et al., 2013).

O C. parvum e C. hominis, responsáveis pela maior parte das infecções humanas (RYAN et al., 2002; XIAO; FENG, 2008), seguidos por $C$. meleagridis (BERRILLI, et al., 2012) podem ter em ovinos importantes reservatórios da infecção zoonótica (XIAO; FAYER, 2008, QUÍLEZ, et al., 2008), uma vez que já foram caracterizados molecularmente nesta espécie animal (XIAO et al., 2004; RYAN et al., 2005; CAMA et al., 2008, ZUCATTO, 2013).

Em um surto de criptosporidiose em uma fazenda de criação mista de gado bovino e ovelhas, no centro da Itália. Um total de 450 ovinos (250 ovelhas e 200 cordeiros) and 140 bovinos (130 vacas e 10 bezerrros) foram mantidos separados na época do surto. Em torno de metade dos cordeiros tiveram diarréia por Cryptosporidium sp. e taxa de mortalidade de $80 \%$, sendo que os bezerros não estavam infectados. The parasite was identified as C.parvum pelo PCR e por análise sequencial pelo gene CpA135. Genotipagem por meio do gene GP60 mostrou a presença do genótipo muito raro, IIaA20G2R1. Logo após, o filho do fazendeiro, com 18 anos, foi hospitalizado com gastroenterite aguda ocasionada pelo mesmo genótipo identificado no surto e este foi o primeiro caso de transmissão zoonótica da criptosporidiose ovina na Itália (CACCIÓ et al., 2013).

\section{Criptosporidiose em Equinos}

A primeira descrição de Cryptosporidium spp. em equinos foi realizada em potros árabes com comprometimento do sistema imune (SNYDER; ENGLAND; MCCHESNEY, 1978). Infecção grave em hospedeiros imunossuprimidos, que podem apresentar diarreia aquosa, má absorção e perda de peso (JOHNSON et al., 1997). Sua manifestação clínica é pouco relatada em equinos, com muitos casos assintomáticos (OLSON et al., 1997; MARQUES, 2010; LAATAMNA et al., 2013).

Em Taquara, município da Região Metropolitana de Porto Alegre, no estado do RS, no Brasil, pela Técnica de Zielh-Nielsen, foi constatada a ocorrência de Cryptosporidium muris (BRACCINI et al., 1993), Cryptosporidium parvum (BRACCINI et al., 1994) e infecções mistas destas duas espécies do parasito (SILVA et al., 1996) em equinos.

Em estudo sobre prevalência de Cryptosporidium spp. utilizando a técnica de RIFI, Johnson et al. (1997) pesquisaram a presença do agente em 91 cavalos adultos provenientes de 16 regiões da Califórnia e não detectaram o protozoário nas amostras avaliadas.

Majewska, Tamang e Graczyk (2004) observaram concordância entre as técnicas de coloração com Ziel-Nielsen e ELISA, verificando ocorrência de $11 \%$ de oocistos de C. parvum em cavalos da Polônia.

Gomes et al. (2008), em Santa Maria, Rio Grande do Sul, verificaram presença de Cryptosporidium spp. em 75\% (48/64) dos equinos estabulados no Jockey Club, por meio da técnica de Faust modificada. Toscan et al. (2010) no mesmo Município, pela mesma técnica, detectaram 38,5\% (20/52) de positividade para Cryptosporidium spp. em cavalos de tração, sem raça definida e 80,8\% em cavalos atletas, da raça Puro Sangue Inglês. Maior prevalência foi evidenciada entre os animais jovens, com elevada ocorrência em cavalos assintomáticos, o que implica em contaminação do meio ambiente e possíveis riscos de infecção, especialmente para os cocheiros.

A prevalência em equinos pode variar conforme o clima, o manejo, a população, localização geográfica, método diagnóstico, idade e outros fatores. A infecção natural por Cryptosporidium 
spp., Giardia spp. e Eimeria leuckarti em equinos com diferentes tipos de manejo, provavelmente está relacionada ao estado imunológico e às condições sanitárias (SOUZA et al., 2009).

Marques (2010) examinaram 90 amostras fecais em Porto Alegre, RS, Brasil, sendo que 71 equinos eram do exército brasileiro, sete do Hospital da Escola de Medicina Veterinária da Universidade Federal do Rio Grande do Sul e 12 de uma estação de treinamento. Por meio da técnica de coloração de Ziehl-Neelsen, a ocorrência de oocistos de Cryptosporidium spp. foi $27.87 \%$ (25/90), sendo verificado positividade apenas em cavalos mantidos no exército e entre os mesmos, 35.21\% (25/71) estavam infectados.

Sevá et al. (2010) em fazendas da região Sudeste do Brasil, examinaram amostras fecais de 63 cavalos pela técnica de centrifugo-flutuação em sacarose (Sheather) e estes apresentaram resultados negativos para este protozoário.

Inacio et al. (2012) observaram ocorrência de Cryptosporidium spp. em 21,8\% (21/101) dos potros e $17,8 \%(18 / 101)$ das éguas pela técnica de Kinyoun. Neste estudo, potros com idade superior dois meses e animais da raça Mangalarga foram menos susceptíveis à esta doença.

Em cinco fazendas na Itália, Veronesi et al. (2010) observaram que 8\% (12/150) dos animais excretavam oocistos do protozoário pela técnica de RIFI, ocorrência comparativamente superior a detectada pelas técnicas de Sheather e ZiehlNeelsen. A caracterização molecular das 12 amostras positivas revelou C. parvum.

Burton et al. (2010) observaram positividade, por meio da técnica da RIFI, para Cryptosporidium spp. em 7,4\% (13/175) dos potros e 1,7\% (3/174) em suas respectivas mães, em 14 fazendas em Nova Iorque (EUA). DNA de oocistos de Cryptosporidium spp. foi detectado em nove potros e em nenhuma das éguas, e o sequenciamento dessas amostras revelou Cryptosporidium genótipo horse.
O genótipo equino de Cryptosporidium (B141) e C. parvum genótipo bovino (B2-8) foram identificados em cavalos da raça Prezwalski's por Ryan et al. (2003). O C. parvum genótipo 2, um agente etiológico envolvido em infecções humanas, foi observado em cavalos domesticados por Chalmers et al. (2005).

Chalmers e Grinberg (2005); Grinberg et al. (2008) isolaram e identificaram por meio de biologia molecular Cryptosporidium spp. em potros com diarreia na Nova Zelândia, para inferir sobre possíveis rotas de transmissão. Estes pesquisadores não concluíram a origem deste parasito ou o seu potencial zoonótico, entretanto, foram identificados diferentes alelos de C. parvum. Os autores sugeriram que este polimorfismo reflete a diversidade genética em C. parvum.

Um estudo realizado por Perrucci et al. (2011) em 37 éguas poucos dias antes e após o parto e em seus respectivos potros apresentou quatro amostras positivas, sendo três de potros e uma de égua; a sequência de nucleotídeos analisada revelou a espécie C. parvum. Quatro animais, na Algéria, foram positivos para o subtipo XIIIa A22R9 do Cryptosporidium (LAATAMNA et al., 2013)

\section{Criptosporidiose em Caninos e Felinos}

Historicamente, Iseki (1979) descreveu o primeiro relato desta infecção em gatos, com detecção de oocistos fecais á microscopia ótica e observação de estágios endógenos na microscopia eletrônica, denominando-o Cryptosporidium felis. Por sua vez, este protozoário foi descrito pela primeira vez em cães por Tzipori e Campbell (1981), sendo reconhecido o primeiro caso clínico de criptosporidiose canina por Wilson, Holscher e Lyle (1983).

O genótipo canino de C. parvum foi designado como uma nova espécie, denominada C. canis (FAYER et al., 2001), com base nos resultados dos experimentos de transmissão cruzada e análises 
genéticas. Estudos moleculares indicaram que cães podem transmitir o genótipo bovino de C. parvum, que é conhecido por ser patogênico, para humanos (ABE et al., 2002).

Relatos de episódios diarreicos com isolamento de C. canis (FAYER et al., 2001), C. felis (CACCIÓ et al., 2002) e C. parvum foram descritos em portadores da síndrome da imunodeficiência adquirida (BALLWEBER et al., 2009). O diagnóstico parasitológico desta infecção deveria ser adotado como um exame laboratorial de rotina na clínica de pequenos animais (MARQUES, 2009).

Apesar das espécies C. parvum, C. felis, $C$. canis, $C$. meleagridis e $C$. muris terem sido isoladas em seres humanos, o risco de infecção humana por contato direto ou indireto com animais de companhia ainda é indeterminado (CACCIÓ et al., 2002; THOMPSON; PALMER; O'HANDLEY, 2008; SMITH et al., 2009).

Apesar da descrição de C. muris em felinos (SANTÍN et al., 2006; FITZGERALD et al., 2011), estes hospedeiros são geralmente infectados por C. felis, cães por C. canis e C. parvum e o ser humano por $C$. hominis e $C$. parvum (HUBER et al., 2007; THOMAZ et al., 2007; BALLWEBER et al., 2009; BOWMAN; LUCIO-FORSTER, 2010; YOSHIUCHI et al., 2010; FITZGERALD et al., 2011).

Em adultos, o estresse pode induzir queda na resistência acarretando no hospedeiro a infecção subclínica crônica com ou sem eliminação de oocistos (NAVARRO et al., 1997; THOMPSON; PALMER; O'HANDLEY, 2008). Com relação à condição imunitária, em animais imunocompetentes, a infecção assume caráter auto-limitante e a detecção deste parasito é mais frequente em animais jovens (YOSHIUCHI et al., 2010).

Cães eliminam oocistos fecais, comumente em infecções crônicas e subclínicas, representando uma potencial fonte de infecção humana (MUNDIM et al., 2007; THOMPSON; PALMER; O'HANDLEY, 2008; SMITH et al., 2009; YOSHIUCHI et al., 2010; WANG et al., 2012). Alguns autores afirmam que cães não representam um risco significativo em termos de saúde pública (ABE et al., 2002; BOWMAN; LUCIO-FORSTER, 2010; UEHLINGER et al., 2013).

Em estudos epidemiológicos da ocorrência de Cryptosporidium spp. na espécie canina, autores relataram desde a ausência (FAYER et al., 2001; SANTOS et al., 2007) com taxas que variam muito e atingem até $44,1 \%$ (FIGUEIREDO et al., 2004; HUBER; BOMFIM; GOMES, 2005; EDERLI; RODRIGUES; CARVALHO, 2005; LALLO; BONDAN, 2006; LABRUNA et al., 2006; HAMNES; GJERDE; ROBERTSON, 2007; KATAGIRI; OLIVEIRA-SEQUEIRA, 2008; MUNDIM et al., 2007; BRESCIANI et al., 2008; MOURA et al., 2009; MANDARINO-PEREIRA et al., 2010; UEHLINGER et al., 2013).

$\mathrm{Na}$ espécie canina, a detecção deste protozoário pode não ser influenciada pela origem urbana ou rural (FIGUEIREDO et al., 2004), por condições ambientais (HUBER; BOMFIM; GOMES, 2005), pela raça (LALLO; BONDAN, 2006), pelo sexo (HUBER; BOMFIM; GOMES, 2005; LALLO; BONDAN, 2006; MUNDIM et al., 2007), mas sim pela ingestão de dieta caseira ou pela manutenção do animal em grama ou terra (MOURA et al., 2009).

Apesar de ter sido verificada maior ocorrência deste coccídio em filhotes (LALLO; BONDAN, 2006; HAMNES; GJERDE; ROBERTSON, 2007; THOMPSON; PALMER; O'HANDLEY, 2008; YOSHIUCHI et al., 2010), algumas pesquisas afirmam que tanto cães jovens como adultos podem estar infectados por Cryptosporidium spp. não sendo verificada diferença significativa em relação à prevalência e à faixa etária dos animais analisados (HUBER; BOMFIM; GOMES, 2005; UEHLINGER et al., 2013).

Gatos domiciliados mostraram-se mais parasitodos por protozoários do que os mantidos em gatis (HUBER; BOMFIM; GOMES, 2002). Em felinos positivos para Cryptosporidium 
foram observadas infecções concomitantes por Toxoplasma gondii, Toxocara cati, Toxoascaris leonina e Cystoisospora spp. e correlação com diarreia (McREYNOLDS et al., 1999; RAMBOZZI et al., 2007).

O Cryptosporidium spp. foi mais comumente encontrado em felinos idosos (McREYNOLDS et al., 1999) ou com idade inferior a um ano (SANTÍN et al., 2006; RAMBOZZI et al., 2007).

Em animais de companhia, a doença clínica foi associada ao desmame, a deficiências nutricionais e a condições de superlotação em canis e/ou gatis (THOMPSON; PALMER; O'HANDLEY, 2008). Entre os endoparasitos, prevaleceu o diagnóstico concomitante dos protozoários Cryptosporidium spp. e Giardia spp. (BALLWEBER et al., 2009).

$\mathrm{Na}$ área rural de Teodoro Sampaio, SP, foi evidenciada infecção por Cryptosporidium spp. em $10,7 \%$ (3/28) dos cães por meio da técnica de Sheather e C.canis foi caracterizado molecularmente (SEVÁ et al., 2010).

\section{Considerações Finais}

$\mathrm{Na}$ literatura brasileira, devido ao alto custo de técnicas moleculares, os métodos baseados em microscopia são os mais utilizados para o diagnóstico de criptosporidiose, sendo a epidemiologia molecular do parasito ainda pouco explorada.

Devido a variedade de hospedeiros e ciclos de transmissão, juntamente à variação genética entre as espécies e genótipos de Cryptosporidium spp., é de fundamental importância a caracterização molecular, a fim de avaliar epidemiologicamente, prevenir e controlar surtos de criptosporidiose em humanos e animais (MEIRELES, 2010; WIDMER et al., 2012).

\section{Referências}

ABE, N.; SAWANO, Y.; YAMADA, K.; KIMATA, I.; ISEKI, M. Cryptosporidium infection in dogs in Osaka,
Japan. Veterinary Parasitology, Amsterdam, v. 108, n. 3, p. 185-93, 2002.

AMER, S.; ZIDAN, S.; FENG, Y.; ADAMU, H., LI, N.; XIAO, L. Identity and public health potential of Cryptosporidium spp. in water buffalo calves in Egypt. Veterinary Parasitology, Amsterdam, v. 191, n. 1-2, p. 123-127, 2013.

AQUINO, M. C. C. Caracterização molecular de Cryptosporidium spp. em bezerros bubalinos do estado de São Paulo, SP. 2012. Dissertação (Mestrado em Ciência Animal) - Universidade Estadual Paulista Julio de Mesquita Filho, Araçatuba.

ARAÚJO, F. A. P.; PAIVA, M. G. S.; ANTUNES, R. L.; CHAPLIN,E. L.; SILVA, N. R. S. Ocorrência de Cryptosporidium parvum e Cryptosporidium muris em búfalos (Bubalus bubalis) no Estado do Amapá, Brasil. Arquivos da Faculdade de Medicina Veterinária, Rio Grande do Sul, v. 24, n. 1, p. 85-90, 1996.

ASSIS, D. C.; RESENDE, D. V.; SANTOS, M. C.; CORREIA, D.; OLIVEIRA-SILVA, M. B. Prevalence and genetic characterization of Cryptosporidium spp. and Cystoisospora belli in HIV-infected patients. Revista do Instituto de Medicina Tropical de São Paulo, São Paulo, v. 55, n. 3, p. 149-154, 2013.

BALLWEBER, L. R.; PANUSKA, C.; HUSTON, C. L.; VASILOPULOS, R.; PHARR, G. T.; MACKIN, A. Prevalence of and risk factors associated with shedding of Cryptosporidium felis in domestic cats of Mississipi and Alabama. Veterinary Parasitology, Amsterdam, v. 160, n. 3-4, p. 306-10, 2009.

BARKER, I. K.; CARBONELL, P. L. Cryptosporidium agni sp. n. from lambs and Cryptosporidium bovis sp. n. from a calf with observations on the oocyst. Parasitology Research, Berlin, v. 44, n. 4, p. 289-298, 1974.

BERRILLI, F., D'ALFONSO, R.; GIANGASPERO, A.; MARANGID, M.; BRANDONISIO, O.; KABOREC, Y.; GLEC, C.; CIANFANELLI, C.; LAURO, R.; DI CAVE, D. Giardia duodenalis genotypes and Cryptosporidium species in humans and domestic animals in Cote d'Ivoire: occurrence and evidence for environmental contamination. Transactions of the Royal Society of Tropical Medicine and Hygiene, London, v. 106, n. 3, p. 191-195, 2012.

BHAT, S. A.; JUYAL, P. D.; SINGLA, L. D. Prevalence of cryptosporidiosis in neonatal buffalo calves in Ludhiana District of Punjab, India. Asian Journal of Animal and Veterinary Advances, India, v. 7, p. 512-520, 2012.

BOMFIM, T. C. B.; HUBER, F.; GOMES, R. S.; ALVES, L. L. Natural infection by Giardia sp. and Cryptosporidium sp. in dairy goats, associated with 
possible risk factors of the studied properties. Veterinary Parasitology, Amsterdam, v. 134, n. 1-2, p. 9-13, 2005.

BOWMAN, D. D.; LUCIO-FORSTER, A. Cryptosporidiosis and giardiasis in dogs and cats: veterinary and public health importance. Experimental Parasitology, New York, v. 124, n. 1, p. 121-7, 2010.

BRACCINI, G. L. E.; SILVA. N. R. S.; CHAPLIN, E. L.; MATTOS, R. C.; ARAUJO, F. A. P. The occurrence of Cryptosporidium muris in Thoroughbred horses in Taquara in Brazil. Arquivos da Faculdade de Veterinária, UFRGS, v. 21, p. 37-42, 1993.

Cryptosporidium parvum in horses from Taquara, RS, Brazil. Arquivos da Faculdade de Veterinária, UFRGS, v. 22, p. 64-69, 1994.

BRESCIANI, K. D. S.; AMARANTE, A. F. T.; LIMA, V. F. M.; FEITOSA, M. M.; FEITOSA, F. L. F.; SERRANO, A. C. M. Infection by Cryptosporidium spp. in dogs from Araçatuba, SP, Brazil: comparison between diagnostic methods and clinical and epidemiological analysis. Veterinária e Zootecnia, Botucatu, v. 15, n. 3, p. 466-8, 2008.

BURTON, A. J.; NYDAM, D. V.; DEAREN, T. K.; MITCHELL, K.; BOWMAN, D. D.; XIAO, L. The prevalence of Cryptosporidium, and identification of the Cryptosporidium horse genotype in foals in New York State. Veterinary Parasitology, Amsterdam, v. 174, n. 1-2, p. 139-144, 2010.

CACCIÓ, S. M.; RINALDI, L.; CRINGOLI, G.; CONDOLEO, R.; POZIO, E. Molecular identification of Cryptosporidium parvum and Giardia duodenalis in the Italian water buffalo (Bubalus bubalis). Veterinary Parasitology, Amsterdam, v. 150, n. 1-2, p. 146-149, 2007.

CACCIÓ, S. M.; SANNELLA, A. R.; MARIANO, V.; VALENTINI, S.; BERTI, F.; TOSINI, F.; POZIO, E. A rare Cryptosporidium parvum genotype associated with infection of lambs and zoonotic transmission in Italy. Veterinary Parasitology, Amsterdam, v. 191, n. 1-2, p. 128-131, 2013.

CACCIÓ, S.; PINTER, E.; FANTINI, R.; MEZZAROMA, I.; POZIO, E. Human infection with Cryptosporidium felis: Case report and literature review. Emerging Infectious Diseases, Atlanta, v. 8, n. 1, p. 85-6, 2002.

CAMA, V. A.; BERN, C.; ROBERTS, J.; CABRERA, L.; STERLING, C. R.; ORTEGA, Y.; GILMAN, R. H.; XIAO, L. Cryptosporidium species and subtypes and clinical manifestations in children, Peru. Emerging Infectious Diseases, Atlanta, v. 14, n. 10, p. 1567-1574, 2008.
CANESTRI-TROTTI, G.; QUESADA, A. First report of Cryptosporidium sp. In Italian water buffalo (Bubalus bubalis). Atti de la Societa Italiana di Scienze Veterinari, Faenza, v. 37, p. 737-740, 1983.

CASTRO-HERMIDA, J. A.; PORS, I.; POUPIN, B.; ARES-MAZÁS, E.; CHARTIER, C. Prevalence of Giardia duodenalis and Cryptosporidium parvum in goat kids in western France. Small Ruminant Research, Amsterdam, v. 56, n. 1, p. 259-264, 2005.

CAUSAPÉ, A. C.; QUILEZ, J.; SANCHEZ-ACEDO, C.; DEL CACHO, C. E.; LÓPEZ-BERNAD, F. Prevalence and analysis of potencial risk factors for Cryptosporidium parvum infection in lambs in Zaragoza (northeastern Spain). Veterinary Parasitology, Amsterdam, v. 104, n. 4, p. 287-298, 2002.

CHALMERS, R. M.; GRINBERG, A. Significance of Cryptosporidium parvum in horses. Veterinary Record, London, v. 156, n. 21, p. 688, 2005.

CHALMERS, R. M.; KATZER, F. Looking for Cryptosporidium: the application of advances in detection and diagnosis. Trends in Parasitology, Oxford, v. 29 , n. 5 , p. 237-251, 2013.

CHALMERS, R. M.; THOMAS, A. L.; BUTLER, B. A.; MOREL, M. C. Identification of Cryptosporidium parvum genotype 2 in domestic horses. Veterinary Record, London, v. 156, n. 2, p. 49-50, 2005.

CIELOSZYK, J.; GONIA, P.; GARCÍA, A.; REMACHAB, M. A.; SÁNCHEZ, E.; CLAVEL, A. Two cases of zoonotic cryptosporidiosis in Spain by the unusual species Cryptosporidium ubiquitum and Cryptosporidium felis. Enfermedades Infecciosas y Microbiología Clínica, Barcelona, v. 2, n. 30, p. 549-551, 2012.

COELHO, W. M. D. Detecção molecular e subtipagem de Cryptosporidium spp. em caprinos, ovinos, bovinos, leitões e equinos jovens. 2011. Tese (Doutorado em Medicina Veterinária Preventiva) - Universidade Estadual Paulista Julio de Mesquita Filho, Jaboticabal.

CONDOLEO, R. U.; RINALDI, L.; SARALLI, G.; MORGOGLIONE, M. E.; SCHIOPPI, M.; CONDOLEO, R.; MUSELLA, V.; CRINGOLI, G. An updating on Cryptosporidium parvum in the water buffalo. Italian Journal of Animal Science, Pavia, v. 6, n. 2, p. 917-919, 2007.

COSENDEY, R. I. J.; FIÚZA, V. R. S.; TEIXEIRA, C. S.; OLIVEIRA, F. C. R. Frequência de oocistos de coccídios do gênero Cryptosporidium em ovinos no estado do Rio de Janeiro. Revista Brasileira de Saúde e Produção Animal, Salvador, v. 9, n. 4, p. 687-695, 2008. 
DECUBELLIS, J.; GRAHAM, J. Gastrointestinal disease in guinea pigs and rabbits. Veterinary Clinics of North America: Exotic Animal Practice, Philadelphia, v. 16, n. 2, p. 421-435, 2013.

DELAFOSSE, A.; CASTRO-HERMIDA, J. A.; BAUDRY, C.; ARES-MAZAS, E.; CHARTIER, C. Herd-level risk factors for Cryptosporidium infection in dairy-goat kids in western France. Preventive Veterinary Medicine, Colorado, v. 77, n. 1-2, p. 109-121, 2006.

DUBEY, J. P.; FAYER, R.; RAO, J. R. Cryptosporidial oocysts in faeces of water buffalo and zebu cattle in India. Journal Veterinary Parasitology, Amsterdam, v. 6, n. 1, p. 55-56, 1992.

EDERLI, B. B.; RODRIGUES, M. F. G.; CARVALHO, C. B. Oocysts of the genus Cryptosporidium in domiciliated dogs from the city of Campos dos Goytacazes, the State of Rio de Janeiro. Revista Brasileira de Parasitologia Veterinária, São Carlos, v. 14, n. 3, p. 129-131, 2005.

EL-GHARI, M. M.; EL-RASHIDY, A. A.; METTIAS, K. N.; HASSEN, E. R.; HASSEN, H. M. Studies on neonatal diarrhea in buffalo calves. In: WORLD BUFFALO CONGRESS, 4., São Paulo, 1994. Proceedings... São Paulo: ABCB, 1994. p. 361-363.

EL-KHODERY, S. A.; OSMAN, S. A. Cryptosporidiosis in buffalo calves (Bubalus bubalis): prevalence and potential risk factors. Tropical Animal Health Production, Edinburgh, v. 40, n. 6, p. 419-426, 2008.

ELWIN, K.; CHALMERS, R. M. Contemporary identification of previously reported novel Cryptosporidium isolates reveals Cryptosporidium bovis and the cervine genotype in sheep (Ovis aries). Parasitology Research, Berlin, v. 102, n. 5, p. 1103-1105, 2008.

FAYER, R. Taxonomy and species delimitation in Cryptosporidium. Experimental Parasitology, New York, v. 124, n. 1, p. 90-97, 2010.

FAYER, R.; SANTÍN, M. Cryptosporidium xiaoi n. sp. (Apicomplexa: Cryptosporidiidae) in sheep (Ovis aries). Veterinary Parasitology, Amsterdam, v. 164, n. 2-4, p. 192-200, 2009.

FAYER, R.; TROUT, J. M.; XIAO, L.; MORGAN, U. M.; LAL, A. A.; DUBEY, J. P. Cryptosporidium canis n.sp. from domestic dogs. Journal Parasitolology, United States, v. 87, n. 6, p. 1415-22, 2001.

FENG, Y.; KARNA, S. R.; DEAREN, T. K.; SINGH, D. K.; ADHIKARI, L. N.; SHRESTHA, A.; XIAO, L. Common occurrence of a unique Cryptosporidium ryanae variant in zebu cattle and water buffaloes in the buffer zone of the Chitwan National Park, Nepal.
Veterinary Parasitology, Amsterdam, v. 185, n. 2-4, p. 309-314, 2012.

FÉRES, F. C.; LOMBARDI, A. L.; CARVALHO, M. P. P.; MENDES, L. C. N.; PEIRÓ, J. R.; CADIOLI, F. A.; MEIRELES, M. V.; PERRI, S. H. V.; FEITOSA, F. L. F. Ocorrência e caracterização molecular de Cryptosporidium em cordeiros. Arquivo Brasileiro de Medicina Veterinária e Zootecnia, Botucatu, v. 61, n. 4, p. 1002-1005, 2009.

FIGUEIREDO, H. C. P.; JUNIOR, D. J. P.; NOGUEIRA, R. B.; COSTA, P. R. S. Excreção de oocistos de Cryptosporidium parvum em cães saudáveis das cidades de Lavras e Viçosa, Estado de Minas Gerais, Brasil. Ciência Rural, Santa Maria, v. 34, n. 5, p. 1625-7, 2004.

FITZGERALD, L.; BENNETT, M.; NG, J.; NICHOLLS, P.; JAMES, F.; ELLIOT, A.; SLAVEN, M.; RYAN, U. Morphological and molecular characterisation of a mixed Cryptosporidium muris/Cryptosporidium felis infection in a cat. Veterinary Parasitology, Amsterdam, v. 175, n. 1-2, p. 160-164, 2011.

FIUZA, V. R. S.; COSENDEY, R. I. J.; FRAZÃOTEIXEIRA, E.; SANTÍN, M.; FAYER, R.; OLIVEIRA, F. C. R. Molecular characterization of Cryptosporidium in Brazilian sheep. Veterinary Parasitolology, Amsterdam, v. 175, n. 3-4, p. 360-362, 2011.

FOREYT, W. J. Coccidiosis and cryptosporidiosis in sheep and goats. Veterinary Clinics of North America: Food Animal Practice, Philadelphia, v. 6, n. 3, p. 655670, 1990.

GAliERO, G.; CONSAlVO, F.; CARUllO, M. La criptosporidiosis nei vitelli bufalini: un aggiornamento. La Selezione Veterinaria, Lombardia, v. 35, n. 5, p. 449453, 1994.

GEURDEN, T.; THOMAS, P.; CASAERT, S.; VERCRUYSSE, J.; CLAEREBOUT, E. Prevalence and molecular characterization of Cryptosporidium and Giardia in lambs and goat kids in Belgium. Veterinary Parasitology, Amsterdam, v. 155, n. 1-2, p. 142-145, 2008.

GOMA, F. Y.; GEURDEN, T.; SIWILA, J.; PHIRI, I. G. K.; GABRIEL, S.; CLAEREBOUT, E.; VERCRUYSSE, J. The prevalence and molecular characterization of Cryptosporidium spp. in small ruminants in Zambia. Small Ruminant Research, Amsterdam, v. 72, n. 1, p. 7780, 2007.

GOMES, A. D.; BARRETA, C.; ZIEGLER, D. P.; SAUSEN, L.; STOEVER, N.; SANGIONI, L. A.; VOGEL, F. S. F.; MONTEIRO, S. G.; ZANELLA, A. Prevalência de Cryptosporidium spp e Giardia sp em equinos estabulados no Jockey Club de Santa Maria - RS, 
Brasil. Ciência Rural, Santa Maria, v. 38, n. 9, p. $2662-$ 2665, 2008.

GOMÉZ-COUSO, H.; AMAR, C. F.; MCLAUCHLIN, J.; ARES-MAZAS, E. Characterisation of a Cryptosporidium isolate from water buffalo (Bubalus bubalis) by sequencing of a fragment of the Cryptosporidium oocyst wall protein gene (COWP). Veterinary Parasitology, Amsterdam, v. 131, n. 1-2, p. 139-144, 2005.

GREEN, R. E.; AMARANTE, A. F. T.; MASCARINI, L. M. Patterns of shedding of cryptosporidial oocysts by ewes and lambs kept indoors. Brazilian Journal of Veterinary Research and Animal Science, São Paulo, v. 42, n. 3, p. 204-209, 2005.

GRINBERG, A.; LEARMONTH, J.; KWAN, E.; POMROY, W.; VILLALOBOS, N. L.; GIBSON, I.; WIDMER, G. Genetic diversity and zoonotic potential of Cryptosporidium parvum causing foal diarrhea. Journal of Clinical Microbiology, Barcelona, v. 46, n. 7, p. 23962398, 2008.

HAMNES, I. S.; GJERDE, B. J.; ROBERTSON, L. J. A longitudinal study on the occurrence of Cryptosporidium and Giardia in dogs during their first year of life. Acta Veterinary Scandinavica, Copenhagen, v. 49, n. 22, p. 1-10, 2007.

HELMY, Y. A.; KRÜCKEN, J.; NÖCKLERD, K.; SAMSON-HIMMELSTJERNAC, G. V.; ZESSINB, K. H. Molecular epidemiology of Cryptosporidium in livestock animals and humans in the Ismailia province of Egypt. Veterinary Parasitology, Amsterdam, v. 193, n. 1-3, p. 15-24, 2013.

HUBER, F.; BOMFIM, T. C. B.; GOMES, R. S. Comparison among infection with Cryptosporidium sp. and Giardia sp. in cats under two breeding systems. Revista Brasileira de Parasitologia Veterinária, São Carlos, v. 11, n. 1, p. 7-12, 2002.

HUBER, F.; BOMFIM, T. C. B.; GOMES, R. S. Comparison between natural infection by Cryptosporidium sp., Giardia sp. in dogs in two living situations in the West Zone of the municipality of Rio de Janeiro. Veterinary Parasitology, Amsterdam, v. 130, p. 1-2, p. 69-72, 2005.

HUBER, F.; SILVA, S.; BOMFIM, T. C. B.; TEIXEIRA, K. R. S.; BELLO, A. R. Genotipic characterization and phylogenetic analysis of Cryptosporidium sp. from domestic animals in Brazil. Veterinary Parasitology, Amsterdam, v. 150, n. 1-2, p. 65-74, 2007.

IMRE, K.; LUCA, C.; COSTACHE, M.; SALA, C.; MORAR, A.; MORARIU, S.; ILIE, M. S.; IMRE, M.; DARABUS, G. Zoonotic Cryptosporidium parvum in Romanian newborn lambs (Ovis aries). Veterinary
Parasitology, Amsterdam, v. 191, n. 1-2, p. 119- 122, 2013.

INACIO, S. V.; BRITO, R. L. L.; ZUCATTTO, A. S.; COELHO, W. M. D.; AQUINO, M. C. C.; AGUIRRE, A. A. R.; PERRI, S. H. V.; MEIRELES, M. V.; BRESCIANI, K. D. S. Cryptosporidiosis in mares and foals of the northwest region of São Paulo State, Brazil. Revista Brasileira de Parasitologia Veterinária, Jaboticabal, v. 21, n. 4, p. 355-358, 2012.

ISEKI, M. Cryptosporidium felis sp. n. (Protozoa: Eimeriorina) from the domestic cat. Japonese Journal Parasitology, Tokyo, v. 28, n. 7, p. 285-307, 1979.

JOHNSON, E.; ATWILL, E. R.; FILKINS, M. E.; KALUSH, J. The prevalence of shedding of Cryptosporidium and giardia spp. based on a single fecal sample collection from each of 91 horses used for backcountry recreation. Journal Veterinary Diagnostic Investigation, USA, v. 9, n. 1, p. 56-60, 1997.

KARANIS, P.; ALDEYARBI, H. M. Evolution of Cryptosporidium in vitro culture. International Journal for Parasitology, Oxford, v. 41, n. 12, p. 1231-1242, 2011.

KATAGIRI, S.; OLIVEIRA-SEQUEIRA, T. C. G. Prevalence of dog intestinal parasites and risk perception of zoonotic infection by dog owners in São Paulo State, Brazil. Zoonoses and Public Health, Berlin, v. 55, n. 8-10, p. 406-413, 2008.

LAATAMNA, A. E.; WAGNEROVÁ, P.; SAK, B.; KVĚTOŇOVÁ, D.; AISSI, M.; ROST, M.; KVÁČ, M. Equine cryptosporidial infection associated with Cryptosporidium hedgehog genotype in Algeria. Veterinary Parasitology, Amsterdam, 2013. (in press).

LABRUNA, M. B.; PENA, H. F. J.; SOUZA, S. L. P.; PINTER, A.; SILVA, J. C. R.; RAGOZO, A. M. A.; CAMARGO, L. M. A.; GENNARI, S. M. Prevalence of endoparasites in dogs from the urban area of Monte Negro Municipality, Rondonia, Brazil. Arquivos do Instituto Biológico, São Paulo, v. 73, n. 2, p. 183-193, 2006.

LALLO, M. A.; BONDAN, E. F. Prevalência de Criptosporidium em cães de instituições da cidade de São Paulo. Revista de Saúde Pública, São Paulo, v. 40, n. 1, p. 120-125, 2006.

MAIKAI, B. V.; BABA-ONOJA, E. B. T.; ELISHA, I. A. Contamination of raw vegetables with Cryptosporidium oocysts in markets within Zaria metropolis, Kaduna State, Nigeria. Food Control, Vurrey, v. 31, n. 1, p. 4548, 2013.

MAJEWSKA, A. C.; TAMANG, P. S. L.; GRACZYK, T. K. Equine Cryptosporidium parvum infections in 
western Poland. Parasitology Research, Berlin, v. 93, n. 4, p. 274-278, 2004.

MAJEWSKA, A. C.; WERNER, A.; SULIMA, P.; LUTY, T. Prevalence of Cryptosporidium in sheep and goats bred on five farms in west-central region of Poland. Veterinary Parasitology, Amsterdam, v. 89, n. 4, p. 269275, 2000.

MANDARINO-PEREIRA, A.; SOUZA, F. S.; DE LOPES, C. W. G.; PEREIRA, M. J. S. Prevalence of parasites in soil and dog feces according to diagnostic tests. Veterinary Parasitology, Amsterdam, v. 170, n. 1-2, p. 176-181, 2010.

MANZANILLA, Y. D. V. C. Excreción de ooquistes de Cryptosporidium spp. y Eimeria spp. en búfalos de dos explotaciones ganaderas del occidente del país. Mundo Pecuário, Los Andes, v. 5, n. 1, p. 64-93, 2009.

MARQUES, S. M. T. Cryptosporidiosis in horses of urban areas of Porto Alegre, Rio Grande do Sul, Southern Brazil. Journal of Equine Veterinary Science, v. 30, n. 7, p. 356-358, 2010.

Diagnosis of Cryptosporidium spp.: should it be part of routine parasitological tests? Veterinária em Foco, Canoas, v. 7, n. 1, p. 28-35, 2009.

MASON, R. W.; HARTLEY, W. J.; TILT, L. Intestinal cryptosporidiosis in a kid goat. The Journal of the Australian Veterinary Association, v. 57, n. 8, p. 386-388, 1981.

McREYNOLDS, C. A.; LAPPIN, M. R.; UNGAR, B.; McREYNOLDS, L. M.; BRUNS, C.; SPILKER, M. M. Regional seroprevalence of Cryptosporidium parvumspecific $\operatorname{IgG}$ of cats in the United States. Veterinary Parasitolology, Amsterdam, v. 80, n. 3, p. 187-95, 1999.

MEIRELES, M. V. Cryptosporidium infection in Brazil: implications for veterinary medicine and public health. Revista Brasileira de Parasitologia Veterinária, São Carlos, v. 19, n. 4, p. 197-204, 2010.

MONIS, P. T.; THOMPSON, R. C. A. Cryptosporidium and Giardia-zoonoses: fact or fiction? Infectious, Genetic and Evolution. Journal of Molecular Epidemiology and Evolutionary Genetics in Infectious Diseases, Irvine, v. 3, n. 4, p. 233-244, 2003.

MOURA, A. B.; TEIXEIRA, E. B.; SOUZA, A. P.; SARTOR, A. A.; BELLATO, V.; STALLIVIERE, F. M. Cryptosporidium spp. em cães domiciliados da cidade de Lages, SC. Revista de Ciências Agroveterinárias, Lages, v. 8, n. 2, p. 173-178, 2009.

MUELLER-DOBLIES, D.; GILES, M.; ELWIN, K.; SMITH, R.P.; CLIFTON-HADLEY, F. A.; CHALMERS, R. M. Distribution of Cryptosporidium species in sheep in the UK. Veterinary Parasitology, Amsterdam, v. 154, n. 3-4, p. 214-219, 2008.

MUNDIM, M. J. S.; ROSA, L. A. G.; HORTÊNCIO, S. M.; FARIA, E. S. M.; RODRIGUES, R. M.; CURY, M. C. Prevalence of Giardia duodenalis and Cryptosporidium spp. in dogs from different living conditions in Uberlândia, Brazil. Veterinary Parasitology, Amsterdam, v. 144, n. 31, p. 356-359, 2007.

NAVARRO, I. T.; KANO, F. S.; OGAWA, L.; FREIRE, R. L.; VIDOTTO, O. Ocorrência de Cryptosporidium SPP em cães com diarréia atendidos no hospital veterinário da Universidade Estadual de Londrina, PR, Brasil. Semina: Ciências Agrárias, Londrina, v. 18, n. 1, p. 23-25, 1997.

NAVARRO-I-MARTINEZ， L.; SILVA， A. J. da; BORNAY-LLINARES, F. J.; MOURA, I. N.; DEL AGUILA, C.; OLEAGA, A.; PIENIAZEK, N. J. Detection and molecular characterization of Cryptosporidium bovis-like isolate from a newborn lamb in Spain. Journal Parasitology, Oxford, v. 93, n. 6, p. 1536-1538, 2007.

NOORDEEN, F.; RAJAPAKSE, R. P.; FAIZAL, A. C.; HORADAGODA, N. U.; ARULKANTHAN, A. Prevalence of Cryptosporidium infection in goats in selected locations in three agroclimatic zones of Sri Lanka. Veterinary Parasitology, Amsterdam, v. 93, n. 2, p. $95-101,2000$.

NOORDEEN, F.; RAJAPAKSEB, R. P. V. J.; HORADAGODAC, N. U.; ABDUL-CAREEMD, M. F.; ARULKANTHAN, A. Cryptosporidium, an important enteric pathogen in goats. A review. Small Ruminant Research, Amsterdam, v. 106, n. 2-3, p. 77- 82, 2012.

OLSON, M. E.; O'HANDLEY, R. M.; RALSTON, B. J.; MCALLISTER, T. A.; THOMPSON, R. C. A. Update on Cryptosporidium and Giardia infections in cattle. Trends in Parasitology, Oxford, v. 20, n. 4, p. 185-191, 2004.

OLSON, M. E.; THORLAKSON, C. L.; DESELLIERS, L.; MORCK, D. W.; MCALLISTER, T. A. Giardia and Cryptosporidium in Canadian farm animals. Veterinary Parasitology, Amsterdam, v. 68, n. 4, p. 375-381, 1997.

ONG, C. S. L.; EISLER, D. L.; ALIKHANI, A.; FUNG, V. W.; TOMLIN, J.; BOWIE, W. R.; ISAAC-RENTON, J. L. Novel Cryptosporidium genotypes in sporadic cryptosporidiosis cases: first report of human infections with a corvine genotype. Emerging Infectious Diseases, Atlanta, v. 8, n. 1, p. 263-268, 2002.

PERRUCCI，S.; BUGGIANI，C.; SGORBINI， M.; CERCHIAI, I.; OTRANTO, D.; TRAVERSA, D. Cryptosporidium parvum infection in a mare and her foal with foal heat diarrhea. Veterinary Parasitology, Amsterdam, v. 182, n. 2-4, p. 333-6, 2011. 
PLUTZER, J.; KARANIS, P. Genetic polymorphism in Cryptosporidium species: An update. Veterinary Parasitology, Amsterdam, v. 165, n. 3-4, p. 187-199, 2009.

PUGH, D. C. Clínica de ovinos e caprinos. São Paulo: Roca, 2004. 513 p.

QUÍlEZ, J.; TORRES, E.; CHALMERS, R. M.; HADFIELD, S. J.; DEL CACHO, E.; SÁNCHEZACEDO, C. Genotype and subtype characterization of Cryptosporidium in lambs and goat kids in Spain. Applied and Environmental Microbiology, Washington D.C, v. 79, n. 19, p. 6026-6031, 2008.

RADOSTITS, O. M.; GAY, C. C.; BLOOD, D. C.; HINCHCLIFF, K. W. Doenças causadas pelos protozoários. In: Clínica veterinária: um tratado de doenças dos bovinos, ovinos, suínos, caprinos e eqüinos. $9^{\mathrm{a}}$ ed. Rio de Janeiro: Guanabara Koogan, 2000. cap. 25, p. 1176-1180.

RAMBOZZI, L.; MENZANO, A.; MANNELLI, A.; ROMANO, S. Prevalence of cryptosporidian infection in cats in Turin and analysis of risk factors. Journal of Feline Medicine and Surgery, Philadelphia, v. 9, n. 5, p. 392-6, 2007.

RIBEIRO, M. G.; LANGONI, H.; JEREZ, J. A.; LEITE, D. S.; FERREIRA, F; GENNARI, S. M. Identification of enteropathogens from buffalo calves with and without diarrhoea in the Ribeira Valley, State of São Paulo, Brazil. Brazilian Journal of Veteterinary Research and Animal Science, São Paulo, v. 37, n. 2, 2000.

RIEUX, A.; PARAUDA, C.; PORSA, I.; CHARTIER, C. Molecular characterization of Cryptosporidium spp. in pre-weaned kids in a dairy goat farm in western France. Veterinary Parasitology, Amsterdam, v. 192, n. 1-3, p. 268-272, 2013.

RINALDI, L.; CONDOLEO, R. U.; CONDOLEO, R.; SARALLI, G.; BRUNI, G.; CRINGOLI, G. Molecular characterization of Cryptosporidium spp. in pre-weaned kids in a dairy goat farm in western France Veterinary Research Communications, Philadelphia, v. 192, n. 1-3, p. 253-255, 2007.

ROBERTSON, L. J.; GJERDE, B. K.; FURUSETH HANSEN, E. The zoonotic potential of Giardia and Cryptosporidium in Norwegian sheep: A longitudinal investigation of 6 flocks of lambs. Veterinary Parasitology, Amsterdam, v. 171, n. 1-2, p. 140-145, 2010.

RODRÍGUEZ-DIEGO, J.; ABREU, J. R.; PÉREZ, E.; ROQUE, E.; CARTAS, O. Presencia de Cryptosporidium sp. en búfalos (Bubalus bubalis) en Cuba. Revista de Salud Animal, La Habana, v. 13, p. 78-80, 1991.
RYAN, U.; XIAO, L.; READ, C.; ZHOU, L.; LAL, A. A.; PAVLASEK, I. Identification of novel Cryptosporidium genotypes from the czech republic. Applied and Environmental Microbiology, Washington D.C, v. 67, n. 7, p. 4302-4307, 2003.

RYAN, U. M.; BATH, C.; ROBERTSON, I.; READ, C.; ELLIOT, A.; MCINNES, L.; TRAUB, R.; BESIER, B. Sheep may not be an important zoonotic reservoir for Cryptosporidium and Giardia parasites. Applied and Environmental Microbiology, Washington D.C, v. 71, n. 9, p. 4992-4997, 2005.

RYAN, U. M.; FALL, A.; WARD, L. A.; HIJJAWI, N.; SULAIMAN, I.; FAYER, R.; THOMPSON, R. C.; OLSON, M.; LAL, A.; XIAO, L. Cryptosporidium hominis n. sp. (Apicomplexa: Cryptosporidiidae) from Homo sapiens. Journal of Eukaryotic Microbiology, Washington, v. 49, n. 6, p. 433-440, 2002.

SANTÍN, M.; TROUT, J. M.; FAYER, R. Prevalence and molecular characterization of Cryptosporidium and Giardia species and genotypes in sheep in Maryland. Veterinary Parasitology, Amsterdam, v. 146, n. 1-2, p. 17-24, 2007.

SANTÍN, M.; TROUT, J. M.; VECINO, J. A. C.; DUBEY, J. P.; FAYER, R. Cryptosporidium, Giardia and Enterocytozoon bieneusi in cats from Bogota (Colombia) and genotyping of isolates. Veterinary Parasitology, Amsterdam, v. 141, n. 3-4, p. 334-9, 2006.

SANTOS, F. A. G.; YAMAMURA, M. H.; VIDOTTO, O.; CAMARGO, P. L. Ocorrência de parasitos gastrintestinais em cães (Canis familiaris) com diarréia aguda oriundos da região Metropolitana de Londrina, Estado do Paraná, Brasil. Semina: Ciências Agrárias, Londrina, v. 28, n. 2, p. 257-268, 2007.

SAVIOLI, L.; SMITH, H.; THOMPSON, A. Giardia and Cryptosporidium join the 'neglected diseases initiative'. Trends in Parasitology, Oxford, v. 22, n. 5 p. 203-208, 2006.

SEVÁ, A. P.; FUNADA, M. R.; SOUZA, S. O.; NAVA, A.; RICHTZENHAIN, L. J.; SOARES, R. M. Occurrence and molecular characterization of Cryptosporidium spp. isolated from domestic animals in a rural area surrounding Atlantic dry forest fragments in Teodoro Sampaio municipality, State of São Paulo, Brazil. Revista Brasileira de Parasitologia Veterinária, São Carlos, v. 19, n. 4, p. 249-253, 2010.

SHARMA, P.; SHARMA, A.; SEHGAL, R.; MALLA, N.; KHURANA, S. Genetic diversity of Cryptosporidium isolates from patients in North India. International Journal of Infectious Diseases, Hamilton, v. 17, n. 8, p. e601-605, 2013. 
SHEN, Y.; YIN, J.; YUAN, Z.; LU, W.; XU, Y.; XIAO, L.; CAO, J. The identification of the Cryptosporidium ubiquitum in preweaned ovines from aba tibetan and qiang autonomous Prefecture in China. Biomedical and Environmental Sciences, San Diego, v. 24, n. 3, p. 315320, 2011.

SILVA, F. M. P. Diagnóstico e caracterização molecular de Giardia duodenalis e Cryptosporidium spp. em amostras fecais de bovinos e ovinos. 2007. Dissertação (Mestrado em Medicina Veterinária) - Faculdade de Medicina Veterinária e Zootécnica da Universidade Estadual Paulista, Botucatu.

SILVA. N. R. S.; BRACCINI, G. L. E.; CHAPLIN, E. L.; ARAUJO, F. A. P. Mixed infection of horses with Cryptosporidium parvum and C. muris in the State of Rio Grande do Sul, Brazil. Arquivos da Faculdade de Veterinaria, UFRGS, v. 24, n. 1, p. 81-84, 1996.

SILVERLÅS, C.; REINECK, H. B.; NÄSLUND, K.; BJÖRKMAN, C. Is there a need for improved Cryptosporidium diagnostics in Swedish calves? International Journal for Parasitology, Oxford, v. 43, n. 2, p. 155-161, 2013.

SMITH, H. V.; CACCIÓ, S. M.; TAIT, A.; MCLAUCHLIN, J.; THOMPSON, A. R. C. Tools for investigating the environment transmission of Cryptosporidium and Giardia infections in humans. Trends in Parasitology, Oxford, v. 22, n. 4, p. 160-167, 2006.

SMITH, H. V.; NICHOLS, R. A. B. Cryptosporidium: detection in water and food. Experimental Parasitology, New York, v. 124, n. 1, p. 61-79, 2010.

SMITH, R. P.; CHALMERS, K. E.; CLIFTONHADLEY, A.; MUELLER-DOBLIES, D.; WATKINS, J.; PAIBA, G. A. Investigation of the role of companion animals in the zoonotic transmission of cryptosporidiosis. Zoonoses Public Health, Berlin, v. 56, n. 1, p. 24-33, 2009.

SNYDER, S. P.; ENGLAND, J. J.; MCCHESNEY, A. E. Cryptosporidiosis in immunodeficient Arabian foals. Veterinary Pathology, USA, v. 15, n. 1, p. 12-17, 1978.

SOLTANE, R.; GUYOT, K.; DEI-CAS, E.; AYADI, A. Prevalence of Cryptosporidium spp. (Eucoccidiorida: Cryptosporidiidae) in seven species of farm animals in Tunisia. Parasite, France, v. 14, n. 4, p. 335-338, 2007.

SOUZA, P. N. B.; BOMFIM, T. C. B.; HUBER, F.; ABBOUD, L. C. S.; GOMES, R. S. Natural infection by Cryptosporidium sp., Giardia sp. and Eimeria leuckarti in three groups of equines with different handlings in Rio de Janeiro, Brazil. Veterinary Parasitology, Amsterdam, v. 160, n. 3-4, p. 327-333, 2009.
SPÓSITO FILHA, E.; FUJII, T. U.; REBOUCAS, M. M. Cryptosporidium spp in cattle and buffaloes from Vale do Ribeira, State of Sao Paulo, Brazil. Arquivos do Instituto Biológico, São Paulo, v. 65, n. 1, p. 97-101, 1998.

SWEENY, J. P. A.; RYAN, U. M.; ROBERTSON, I. D.; YANG, R.; BELL, K.; JACOBSON, C. Longitudinal investigation of protozoan parasites in meat lamb farms in southern Western Australia. Preventive Veterinary Medicine, Colorado, v. 101, n. 3-4, p. 192-203, 2011.

THOMAZ, A.; MEIRELES, M. V.; SOARES, R. M.; PENA, H. F. J.; GENNARI, S. M. Molecular identification of Cryptosporidium spp. from fecal samples of felines, canines and bovines in the state of São Paulo, Brazilian Veterinary Parasitology, Amsterdam, v. 150, n. 4, p. 291-6, 2007.

THOMPSON, R. C. A.; PALMER, C. S.; O'HANDLEY, $\mathrm{R}$. The public health and clinical significance of Giardia and Cryptosporidium in domestic animals. The Veterinary Journal, London, v. 177, n. 1, p. 18-25, 2008.

TOSCAN, G.; PEREIRA, R. C. F.; VOGEL, F. S. F.; SANGIONI, L. A. Cryptosporidium spp. in traction horse in Santa Maria, RS, Brasil. Arquivo Brasileiro de Medicina Veterinária e Zootecnia, Belo Horizonte, v. 62, n. 1, p. 211-213, 2010.

TZIPORI, S.; CAMPBELL, I. Prevalence of Cryptosporidium antibodies in 10 animal species. Journal of Clinical Microbiology, Barcelona, v. 14, n. 4, p. 455-456, 1981.

UEHLINGER, F. D.; GREENWOOD, S. J.; MCCLURE, J. T.; CONBOY, G.; O'HANDLEY, R.; BARKEMA, H. W. Zoonotic potential of Giardia duodenalis and Cryptosporidium spp. and prevalence of intestinal parasites in young dogsfrom different populations on Prince Edward Island, Canada. Veterinary Parasitology, Amsterdam, v. 196, n. 3-4, p. 509-514, 2013.

VENTURINI, L.; BACIGALUPE, D.; BASSO, W.; UNZAGA, J. M.; VENTURINI, M. C.; MORÉ, G. Cryptosporidium parvum em animales domésticos y em monos de un zoológico. plogía Latinoamericana, v. 61, n. 1-2, p. 90-93, 2006.

VENU, R.; LATHA, B. R.; ABDUL BASITH, S.; DHINAKAR RAJ, G.; SREEKUMAR, S.; RAMAN, M. Molecular prevalence of Cryptosporidium spp. in dairy calves in Southern states of India. Veterinary Parasitology, Amsterdam, v. 188, n. 1-2, p. 19-24, 2012.

VERONESI, F.; PASSAMONTI, F.; CACCIO, S.; DIAFERIA, M.; FIORETTI, D. P. Epidemiological survey on equine Cryptosporidium and Giardia infections in Italy and molecular characterization of isolates. Zoonoses and Public Health, Berlin, v. 57, n. 7-8, p. 510-517, 2010. 
VIEIRA, L. S. Surto de Criptosporidiose em caprinos lactentes. Sobral: Embrapa Caprinos, 1999. 2 p.

VIEIRA, L. S.; SILVA, M. B. O.; TOLENTINO, A. C. V.; LIMA, J. D.; SILVA, A. C. Outbreak of cryptosporidiosis in dairy goats in Brazil. Veterinary Record, London, v. 140, n. 16, p. 427-428, 1997.

WANG, A.; RUCH-GALlIE, R.; SCORZA, V.; LIN, P.; LAPPIN, M. R. Prevalence of Giardia and Cryptosporidium species in dog park attending dogs compared to non-dog park attending dogs in one region of Colorado. Veterinary Parasitology, Amsterdam, v. 184, n. 2-4, p. 335-340, 2012.

WANG, Y.; FENG, Y.; CUI, B.; JIAN, F.; NING, C.; WANG, R.; ZHANG, L.; XIAO, L. Cervine genotype is the major Cryptosporidium genotype in sheep in China. Parasitology Research, Berlin, v. 106, n. 2, p. 341-347, 2010.

WIDMER, G.;YONGSUN,L.;HUNT,P.; MARTINELLI, A.; TOLKOFF, M. BODI, K. Comparative genome analysis of two Cryptosporidium parvum isolates with different host range. Infection, Genetics and Evolution, Amsterdam, v. 12, n. 6, p. 1213-1221, 2012.

WILSON, R. B.; HOLSCHER, M. A.; LYLE, S. J. Cryptosporidiosis in a pup. Journal of American Veterinarian Medical Association, Ithaca, v. 183, n. 9, p. 1005-1006, 1983.

XIAO, L. Molecular epidemiology of cryptosporidiosis: an update. Experimental Parasitology, Amsterdã, v. 124, n. 1, p. 80-89, 2010.
XIAO, L.; FAYER, R. Molecular characterisation of species and genotypes of Cryptosporidium and Giardia and assessment of zoonotic transmission. International Journal Parasitology, Oxford, v. 38, n. 11, p. 1239-1255, 2008.

XIAO, L.; FAYER, R.; RYAN, U.; UPTON, S. J. Cryptosporidium taxonomy: recent advances and implications for public health. Clinical Microbiology Reviews, Washington, v. 17, n. 1, p. 72-97, 2004.

XIAO, L.; FENG, Y. Zoonotic cryptosporidiosis. FEMS Immunology and Medical Microbiology, Amsterdam, v. 52, n. 3, p. 309-323, 2008.

YANG, R.; JACOBSON, C.; GORDON, C.; RYAN, $\mathrm{U}$. Prevalence and characterization of Cryptosporidium and Giardia species in pre-weaned sheep in Austrália. Veterinary Parasitology, Amsterdam, v. 161, n. 1-2, p. 19-24, 2009.

YOSHIUCHI, R.; MATSUBAYASHI, M.; KIMATA, I.; FURUYA, M.; TANI, H.; SASAI, K. Survey and molecular characterization of Cryptosporidium and Giardia spp. in owned companion animal, dogs and cats, in Japan. Veterinary Parasitology, Amsterdam, v. 174, n. 3-4, p. 313-316, 2010.

ZUCATTO, A. S. Ocorrência de parasitoses gastrointestinais em cordeiros no município de Alambari, São Paulo. 2013. Dissertação (Mestrado em Ciência Animal) - Universidade Estadual Paulista Julio de Mesquita Filho, Araçatuba. 